\title{
Metachronous adrenal metastasis from ovarian cancer - a case report and literature review
}

\author{
Nicolae BACALBASA ${ }^{1,2,3}$, Camelia DIACONU ${ }^{1,4}$, Laura ILIESCU ${ }^{1,5}$, Cristian BALALAU ${ }^{1,6}$, \\ Mihai DIMITRIU ${ }^{1,7}$, Cornel SAVU ${ }^{1,8}$, Ovidiu Gabriel BRATU ${ }^{1,9}$, Simona DIMA ${ }^{3}$, \\ Alexandru FILIPESCU ${ }^{1,10}$, Dragos CRETOIU ${ }^{1,11}$, Irina BALESCU ${ }^{12}$ \\ 1"Carol Davila" University of Medicine and Pharmacy, Bucharest, Romania \\ 2Department of Obstetrics and Gynecology, "I. Cantacuzino" Clinical Hospital, Bucharest, Romania \\ ${ }^{3}$ Department of Visceral Surgery, Center of Excellence in Translational Medicine \\ Fundeni Clinical Institute, Bucharest, Romania \\ ${ }^{4}$ Department of Internal Medicine, University Emergency Hospital Bucharest, Bucharest, Romania \\ ${ }^{5}$ Department of Internal Medicine, Fundeni Clinical Institute, Bucharest, Romania \\ ${ }^{6}$ Department of Surgery, Pantelimon Clinical Hospital, Bucharest, Romania \\ ${ }^{7}$ Department of Obstetrics and Gynecology, \\ "Sf. Pantelimon" Emergency Clinical Hospital, Bucharest, Romania \\ ${ }^{8}$ Department of Thoracic Surgery, "Marius Nasta" Institute of Pneumonology Bucharest, Romania \\ ${ }^{9}$ Department of Urology, Emergency Central Military Hospital, \\ Academy of Romanian Scientists, Bucharest, Romania \\ ${ }^{10}$ Department of Obstetrics and Gynecology, Elias Emergency Hospital, Bucharest, Romania \\ 11"Alessandrescu-Rusescu" National Institute of Mother and Child Health, \\ Fetal Medicine Excellence Research Center, Bucharest, Romania \\ ${ }^{12}$ Department of Surgery, Ponderas Academic Hospital, Bucharest, Romania
}

\begin{abstract}
Hematogenous path remains an important pathway of spread for ovarian cancer; the most commonly reported sites of involvement are represented by liver and lungs. The aim of this paper is to report a rare situation in which adrenal metastasis from ovarian carcinoma was encountered.

Case report. The 55-year-old patient with previous history of surgically treated ovarian cancer was diagnosed at 18 months follow-up with an isolated lesion at the level of the right adrenal gland. After completing the biochemical analysis, in order to exclude an endocrine disorder, the final suspicion of diagnostic was of an adrenal metastasis and was submitted to adrenalectomy. The histopathological studies confirmed the presence of an adrenal metastasis of ovarian cancer.

Conclusions. Although rare situations, adrenal metastases from ovarian cancer can be encountered and might be successfully treated by surgery with curative intent.
\end{abstract}

Keywords: adrenal metastases, metachronous, ovarian cancer 


\section{INTRODUCTION}

Ovarian cancer is still considered as a veritable silent killer due to the fact that most patients remain asymptomatic for a long period of time; meanwhile malignant cells present an increased capacity of spread via peritoneal, hematogenous and lymphatic pathways leading to the development of distant metastases (1-3). In such cases surgery remains the option of choice only if radical procedures to no visible residual disease is feasible. Unfortunately this desiderate is rather difficult to be achieved especially in cases in which hematogenous spread is present, in such patients the only rationale therapeutic strategy consisting of systemic palliative treatment. However, in certain cases, isolated metastases are encountered, and the patients might become candidates for surgery with curative intent (3). In such cases significant benefit of survival is to be expected. The aim of the current paper is to report the case of a 55-year-old patient who was diagnosed with an isolated right adrenal metastasis from ovarian cancer, which was successfully submitted to radical surgery.

\section{CASE PRESENTATION}

The 55-year-old female had been previously investigated for diffuse abdominal pain and weight loss and was diagnosed with stage IIIC ovarian cancer two years ago; at the time of diagnostic the presence of distant hematogenous metastases was excluded by the imagistic studies while the paraclinical tests revealed a significant increase of the cancer antigen CA125 levels (at $3,120 \mathrm{U} / \mathrm{ml}$ ).

At that time total hysterectomy en bloc with bilateral adnexectomy, pelvic and para-aortic lymph node dissection, omentectomy, pelvic and parietal peritonectomy were performed. The histopathological studies confirmed the presence of an well differentiated serous ovarian adenocarcinoma with two positive pelvic lymph nodes at the level of the left obturatory fossa and one at the level of the right external iliac artery group (out of the 21 retrieved nodes) and no positive para-aortic lymph node. However, at that moment, venous thrombus at the level of the right ovarian veins was encountered. At four weeks follow-up, the serum levels of CA125 decreased at $23 \mathrm{U} / \mathrm{ml}$, and the patient was submitted to adjuvant chemotherapy consisting of six cycles of taxanes and platinum based chemotherapy. However, 18 months after ending the adjuvant chemotherapy, the patient was diagnosed at computed tomography with a suspect lesion at the level of the right adrenal gland measuring $4.5 \times 4 \times 5$ $\mathrm{cm}$ in association with a slight increase of the serum CA125 (at $145 \mathrm{U} / \mathrm{ml}$ ). The initial suspicion was the one of an isolated metastasis from ovarian cancer; however, due to the rarity of this scenario, the patient was further submitted to a positron emission tomography and to endocrine studies. The imagistic studies excluded the presence of any other active sites and confirmed the presence of a metabolic active lesion at the level of the right adrenal gland while the paraclinical endocrine tests excluded a primary adrenal tumor. The patient was further submitted to surgery, right adrenalectomy being performed (Figures 1, 2). The histopathological studies confirmed the presence of an adrenal metastasis from ovarian cancer. The patient was discharged in the fourth postoperative day; meanwhile, at one month follow-up, the serum levels of CA125 decreased at $19 \mathrm{U} / \mathrm{ml}$, therefore demonstrating the completeness of cytoreduction.

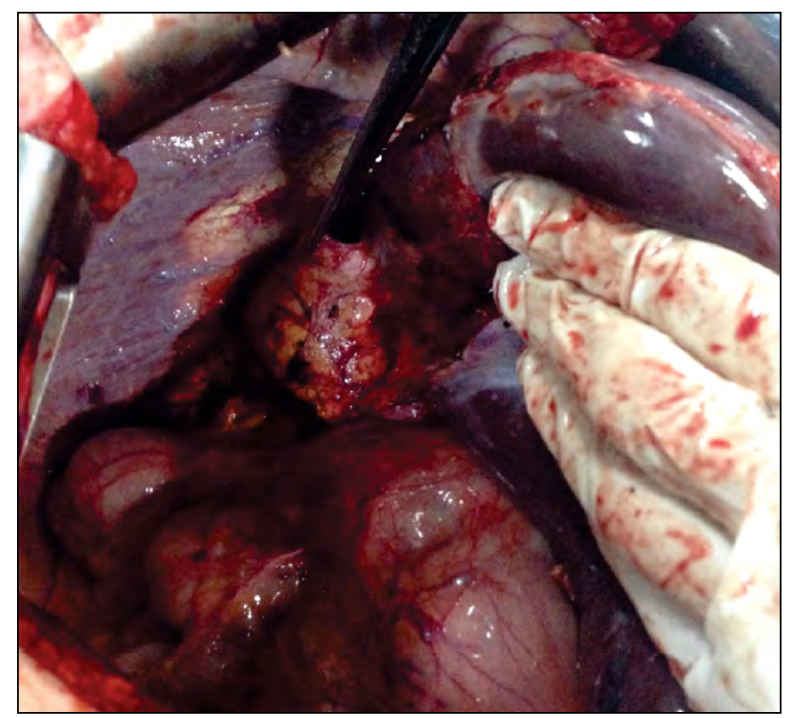

FIGURE 1. Intraoperative aspect - dissection of the right adrenal gland

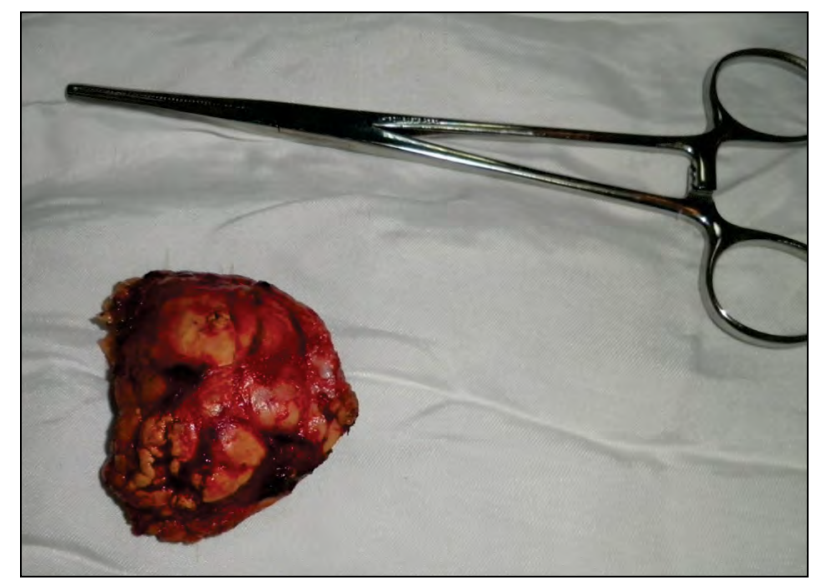

FIGURE 2. The specimen of right adrenalectomy

\section{DISCUSSION}

Adrenal metastases are rarely encountered, the most commonly primaries which lead to the appari- 
tion of secondary tumors at this level being represented by lung and kidney (4). In a study conducted by Castillo et al. on 32 patients submitted to adrenalectomy for metastatic disease, the most commonly encountered primaries were represented by lung carcinoma (in 13 cases), renal cell carcinoma (in 9 cases), colorectal and bladder carcinoma (each in two cases) and ovarian, breast, gastric cancer and melanoma (each in one case). However, autopsy studies came to demonstrate that up to $15 \%$ of ovarian cancer patients will develop adrenal metastases; these findings were revealed at necropsy and were explained by the hematogenous and lymphatic spread of the malignant cells (5). Interestingly, certain authors came to demonstrate that in cases presenting lymph node metastases on a certain side, adrenal gland metastases usually develop in the contralateral adrenal gland side $(1,6,7)$; however, this mechanism of spread is not fully understood so far (1). When it comes to the case we reported, the mechanism of development of an adrenal metastasis might be a complex one, both hematogenous and lymphatic being incriminated. Therefore, the patient associated both hematogenous spread at the level of the ovarian veins and lymphatic spread - at the level of three retrieved pelvic lymph nodes.

As for the symptomatology associated by the presence of adrenal metastases, most patients will remain asymptomatic for a long period of time; meanwhile these patients might become symptomatic in rare situations, especially if bilateral involvement is encountered. An interesting such case was reported by the study group conducted by Gokosmanoglu et al. in 2016 (8); the authors presented the case of a 60-yearold female who was diagnosed with a suspect pelvic mass in association with bilateral adrenal tumors which were initially considered as non-functional adrenal tumors. The patient was submitted to surgery for the gynecological disorder which proved to be an undifferentiated ovarian carcinoma with lymph node metastases; postoperatively the patient developed phenomena of adrenal insufficiency while the adrenal masses proved to be adrenal metastases from ovarian cancer. However, the fulminant development of postoperative adrenal insufficiency was explained by the authors to be caused by the association of postoperative stress in association with tumor growth produced by the immune suppression caused by surgery itself (8).

Most often the presence of adrenal metastases is the sign of systemic neoplastic impregnation and is associated with other disseminated metastases $(2,3,9$ $11)$; in such cases the patient becomes the candidate for systemic, palliative oncological treatment; however, in rare cases isolated lesions are encountered $(12,13)$. When it comes to the most appropriate thera- peutic option in such cases, it seems that surgery remains the option of choice especially in cases with unilateral adrenal metastases; in such cases unilateral adrenalectomy provides a radical procedure which increases the chances of achieving a long term survival. However, this approach should be avoided in cases presenting bilateral lesions or a poor biological status due to the increased risks of postoperative complications $(1,14,15)$.

The first successful resection of an isolated metachronous adrenal metastasis from ovarian cancer was reported in 2002; at that moment Einat et al. presented the case of a patient who was diagnosed with a solitary adrenal metastasis from ovarian cancer 11 months after completing the adjuvant chemotherapy and who was successfully submitted to laparoscopic adrenalectomy; similarly to our case, the serum levels of CA125 reported a significant decrease (from $365 \mathrm{U} /$ $\mathrm{ml}$ to $35 \mathrm{U} / \mathrm{ml}$ ) therefore demonstrating the completeness of cytoreduction (12).

As for the prognostic factors after adrenalectomy for metastatic disease, one of the largest studies conducted on this issue which was published in 2010 by Muth et al. came to demonstrate that patients submitted to surgery with curative intent in the absence of other previous surgery for metastatic disease as well as those presenting colorectal or renal cell carcinoma primaries seemed to have a significantly improved long term survival. Interestingly, no correlation could be established between the size of metastases and overall survival (16). In a more recent study conducted by Tomasini et al. the authors underlined the fact that the origin of the primary tumor does not significantly influence the long term outcomes after adrenalectomy for metastatic disease and recommended performing the surgical procedure whenever oligometastatic metachronous lesions are found. In Tomasini's study the authors included 40 cases with oligometastatic disease, the overall survival being significantly improved in cases presenting metachronous lesions (10.8 months) when compared to synchronous lesions (4.5 months, $p=0.008$ ) (17).

\section{CONCLUSIONS}

Although rarely encountered, adrenal metastases from ovarian cancer should be suspected in patients with a previous history of this malignancy. Due to the rarity of cases, in such patients the initial diagnostic tests should be conducted in order to exclude a primary adrenal pathology; once the differential diagnostic with all these pathologic entities is established, surgery consisting of adrenalectomy should be performed in order to remove the isolated metastasis developed at this level and to achieve a radical surgical gesture. 


\section{Authors' contributions}

NB performed the surgical procedures; IB prepared the manuscript; $C D, L I, D C$, SD performed data analysis; $\mathrm{OGB}, \mathrm{AF}, \mathrm{CB}, \mathrm{MD}, \mathrm{CS}$ were part of the surgical team; $D C$ advised about the oncological outcome; NB revised the final draft of the manuscript.

\section{Acknowledgement}

This work was supported by the project entitled „Multidisciplinary Consortium for Supporting the Research Skills in Diagnosing, Treating and Identifying Predictive Factors of Malignant Gynecologic Disorders", project number PN-III-P1-1.2-PCCDI2017-0833.

\section{REFERENCES}

1. Tokue H, Tokue A, Tsushima Y. Synchronous Adrenal Metastasis and an Inferior Vena Cava Tumor Thrombus From an Ovarian Carcinoma. J Ovarian Res. 2014;7:5.

2. Bacalbasa N, Balescu I, Dima S, Brasoveanu V, Popescu I. Hematogenous Splenic Metastases as an Independent Negative Prognosis Factor at the Moment of Primary Cytoreduction in Advanced Stage Epithelial Ovarian Cancer - A Single Center Experience. Anticancer Res. 2015;35:5649-5654.

3. Bacalbasa N, Balescu I, Dima S, Brasoveanu V, Popescu I. Splenectomy as Part of Cytoreductive Surgery in Recurrent Epithelial Ovarian Cancer. Anticancer Res. 2015;35:5097-5101.

4. Kim SH, Brennan MF, Russo P, Burt ME, Coit DG. The Role of Surgery in the Treatment of Clinically Isolated Adrenal Metastasis. Cancer 1998;82:389-394.

5. Dvoretsky PM, Richards KA, Angel $\mathrm{C}$ et al. Distribution of Disease at Autopsy in 100 Women With Ovarian Cancer. Hum Pathol. 1988;19:57-63.

6. Negishi H, Takeda M, Fujimoto T et al. Lymphatic Mapping and Sentinel Node Identification As Related to the Primary Sites of Lymph Node Metastasis in Early
Stage Ovarian Cancer. Gynecol Oncol. 2004;94:161-166.

7. Cass I, Li AJ, Runowicz CD et al. Pattern of Lymph Node Metastases in Clinically Unilateral Stage I Invasive Epithelial Ovarian Carcinomas. Gynecol Oncol. 2001;80:56-61.

8. Gokosmanoglu F, Varım C, Ergenc H, Yaylaci S, Colak R. Adrenal metastasis stemming from ovarian cancer presented with adrenal insufficiency: A case report and review of the literature. European Journal of Pharmaceutical and Medical Research 2013;3:29-30.

9. Bacalbasa N, Balescu I, Dima S, Popescu I. Ovarian sarcoma carries a poorer prognosis than ovarian epithelial cancer throughout all FIGO stages: a single-center case-control matched study. Anticancer Res. 2014;34:7303-7308.

10. Bacalbasa N, Taras C, Orban C et al. Atypical Right Hepatectomy for Liver Metastasis from Ovarian Leiomyosarcoma - A Case Report and Literature Review. Anticancer Res. 2016;36:1835-1840.

11. Bacalbasa N, Balescu I, Dima S, Popescu I. Long-Term Survivors After Liver Resection for Ovarian Cancer Liver Metastases. Anticancer Res. 2015;35:69196923.
12. Einat S, Amir S, Silvia M, Moshe I. Successful Laparoscopic Removal of a Solitary Adrenal Metastasis From Ovarian Carcinoma: A Case Report. Gynecol Oncol. 2002;85:201-203.

13. Luketich JD, Burt ME. Does Resection of Adrenal Metastases From Non-Small Cell Lung Cancer Improve Survival? Ann Thorac Surg. 1996;62:1614-1616.

14. Sundersingh S, Rajasundaram S, Majhi U. Bilateral adrenal metastases from bilateral small cell neuroendocrine carcinoma of the ovary. Indian J Surg. 2003;65:373-375.

15. Folli S, Zaccaroni A, Mengozzi M, Dell'Amore D, Vio A.Surgical Treatment of Adrenal Metastases. Personal Experience. Minerva Chir. 1998;53:1035-1038.

16. Muth A, Persson F, Jansson S, Johanson V, Ahlman H, Wangberg B. Prognostic Factors for Survival After Surgery for Adrenal Metastasis. Eur J Surg Oncol. 2010;36:699704.

17. Tomasini P, Garcia ME, Greillier L, Paladino C, Sebag F, Barlesi F. Adrenal Surgery for Oligometastatic Tumors Improves Survival in Selected Cases. J Visc Surg. 2017; $154: 87-91$ 